\title{
Discrete self-oscillation period branches observed in semiconductor superlattices
}

\author{
Jun Wang (王军), ${ }^{1,4}{ }^{*}$ Bambi Hu (胡斑比), ${ }^{2,4}$ Zhigang Zheng (郑志刚), ${ }^{3}$ and Zhigang Li (李志刚) ${ }^{1}$ \\ ${ }^{1}$ Department of Mechanical Engineering, The Hong Kong University of Science and Technology, Clear Water Bay, \\ Kowloon, Hong Kong, China \\ ${ }^{2}$ Department of Physics, University of Houston, Houston, Texas 77204-5005, USA \\ ${ }^{3}$ Department of Physics and the Beijing-Hong Kong-Singapore Joint Centre for Nonlinear and Complex Systems (Beijing), \\ Beijing Normal University, Beijing 100875, China \\ ${ }^{4}$ Centre for Nonlinear Studies and the Beijing-Hong Kong-Singapore Joint Centre for Nonlinear and Complex Systems (Hong Kong), \\ Hong Kong Baptist University, Kowloon Tong, Hong Kong, China
}

(Received 1 November 2010; revised manuscript received 9 February 2011; published 8 April 2011)

\begin{abstract}
We investigate the self-sustained current oscillation of a weakly coupled semiconductor superlattice in the dynamical voltage band using a microscopic sequential tunneling model. With the voltage as a control parameter, two types of branches of current oscillation period versus voltage have been observed, which correspond to various oscillation scenarios. The first branch type consists of a series of period branches in accordance with how many charge dipoles need to be created at the emitter side to trigger a dipole-tripole oscillation scenario. For the second branch type, charge dipoles are generated periodically at the emitter, but all of them fail to develop completely and die out, thereby leading to a low-period oscillation scenario without the dipole-tripole process. The bistability between different branches is also observed by voltage up-sweeping and down-sweeping.
\end{abstract}

DOI: 10.1103/PhysRevB.83.155306

PACS number(s): 73.63.-b, 73.50.Fq, 05.45.-a, 73.23.-b

\section{INTRODUCTION}

Nonlinear vertical charge transport in weakly coupled semiconductor superlattices (SLs) has been a research highlight in the interdisciplinary research field between semiconductor physics and nonlinear science during the past years. ${ }^{1-4}$ For weakly coupled doped SLs, the main mechanism of vertical charge transport is sequential tunneling between adjacent SL quantum wells. ${ }^{5-11}$ A great richness of nonlinear transport behaviors has been observed in weakly coupled SLs, including the formation of stationary electrical field domains (EFDs), ${ }^{12-20}$ self-sustained current oscillations (traveling EFDs), ${ }^{21-27}$ and driven and undriven chaos. ${ }^{28-33}$ These dynamic behaviors depend on the applied voltage, SL configuration, boundary conditions, doping density, sample temperature, and other control parameters. Among them, the voltage $V$ and boundary contact conductivity $\sigma$ are two crucial parameters for the transport scenario to be observed.

The $V-\sigma$ phase diagram has been shown in Ref. 27. For sufficiently low $\sigma$, stationary $I-V$ characteristics could be established with a wide charge depletion layer (CDL) as an electric field domain wall which separates the high and low electric field domains. ${ }^{15}$ With increasing $\sigma$, the SL system enters into the oscillation regime via a Hopf bifurcation (lower threshold) and it gives rise to various oscillation modes. ${ }^{33}$ For very large $\sigma$, the system is in the stationary regime again, and the electric field domain wall becomes a charge accumulation layer (CAL). This upper $\sigma$ boundary (upper threshold) between the oscillation and stationary regimes is a sawtooth-like boundary with several tongues, where each tongue corresponds to a specific position of the stationary domain wall. ${ }^{27}$ If the contact conductivity $\sigma$ is fixed at the tongue regime and the voltage $V$ is varied to pass through the tongues one by one, the SL system will be alternately in the stationary and oscillation regimes. As a result, the so-called dynamic voltage bands (DVBs) and static voltage bands (SVBs) will be observed alternately with increasing voltage.
DVBs were first observed in the temperature-induced transition process from stationary $I-V$ characteristics to selfsustained oscillations. ${ }^{34}$ In previous works, ${ }^{34-40}$ the influences of the system temperature and external ac bias on $I-V$ characteristics in the DVB region have been fully investigated. With increasing temperature, the DVBs will emerge at the low voltage side of the SVBs, then expand, and finally squeeze out the SVBs. ${ }^{34,35}$ If ac bias condition is applied, the $I-V$ characteristic curve in the DVB region becomes discrete, which is related to a period-adding bifurcation. ${ }^{39}$

However, few papers focus on the voltage dependence of the oscillation period in the DVB region, except the works in Refs. 35,38,40, where the oscillation frequency was found to increase first and then decrease with increasing voltage. In the present work, we investigate the voltage dependence of the current oscillation period in the DVB region in a microscopic resonant tunneling model. Our simulation results show that the period-voltage curve consists of many distinct branches, which have not been revealed in previous works. We attribute these branches to various dipole generation processes at the emitter side of the SLs.

The rest of this paper is organized as follows: The dimensionless sequential resonant tunneling model and numerical method are briefly described in Sec. II. In Sec. III, we show the voltage dependence of the self-oscillation period in the DVB region. Section IV is devoted to the dipole generation process in order to characterize the discrete period branches. In Sec. V, the hysteresis phenomenon between different period branches is reported by voltage up-sweeping and down-sweeping. Finally, we finish the paper with our conclusions and discussion in Sec. VI.

\section{DIMENSIONLESS MODEL}

Weakly coupled superlattices can be described by a onedimensional discrete sequential tunneling model, which has been developed over the past decades and can be used to model the experimental results quite well (see Refs. 1-4 and 
references cited therein). The framework of this model is based on three well separated time scales: inter-subband scattering time $\tau_{s}$, tunneling time $\tau_{t}$ (the average time an electron needs to escape from one SL well to the adjacent one), and macroscopic time of self-sustained oscillations $\tau_{m}$. Physically, $\tau_{s} \ll \tau_{t} \ll \tau_{m} .{ }^{1,9,13-15}$ Therefore, the electrons in one quantum well have enough time to fall into the first subband from an excited subband before they tunnel to the next well and they are in quasiequilibrium with Fermi-Dirac distribution at the given SL well. This implies that the tunneling current can be considered a quasistationary process on the macroscopic time scale $\tau_{m}$ and the dominant mechanism of vertical charge transport is sequential resonant tunneling.

Following the pioneering works on the sequential resonant tunneling model, we give a brief description about the model and adopt the dimensionless formulas proposed by Bonilla et al. in Ref. 13. All the physical quantities used below are in the dimensionless form if not otherwise indicated. The definitions of the units used to nondimensionalize the model equations and their values for the typical $9 \mathrm{~nm} / 4 \mathrm{~nm}$ GaAs/AlAs superlattices at $T=5 \mathrm{~K}$ can be found in Tables I and II in Ref. 13.

The dynamic equation of the dimensionless electric field $E_{i}$ at well $i$ is given by Ampere's law:

$$
\frac{d E_{i}}{d t}+J_{i \rightarrow(i+1)}=J(t), \text { for } \quad i=0, \ldots, N,
$$

where $J_{i \rightarrow(i+1)}$ represents the tunneling current from well $i$ to well $i+1$ and $N$ is the total number of quantum wells in the superlattices. In this work, $N=40$. Well 0 and $N+1$ denote the charge emitter and collector contact regions, respectively. The tunneling current $J_{i \rightarrow(i+1)}\left(E_{i}, n_{i}, n_{i+1}\right)$ depends on the dimensionless electric field $E_{i}$ at local well $i$ and the electron densities $n_{i}$ and $n_{i+1}$ in the corresponding SL wells. $n_{i}$ and $E_{i}$ are coupled by Poisson's equation,

$$
n_{i}=\frac{E_{i}-E_{i-1}}{v}+1, \text { for } i=1, \ldots, N,
$$

where $v(v=5.212)$ is the dimensionless doping density. To make these equations solvable, the total bias condition,

$$
\sum_{i=0}^{N} E_{i}=(N+1) \phi,
$$

is required, where $(N+1) \phi$ denotes the dimensionless voltage applied to the superlattices.

The calculation of the tunneling current $J_{i \rightarrow(i+1)}$ has been performed by the transfer Hamiltonian method ${ }^{1,9,13}$ and also by a microscopic tunneling Hamiltonian. ${ }^{15}$ In this work, we use the same formulas and parameters as those provided in Ref. 13 for the typical $9 \mathrm{~nm} / 4 \mathrm{~nm} \mathrm{GaAs} / \mathrm{AlAs}$ superlattices. As for the boundary tunneling current $J_{0 \rightarrow 1}$ and $J_{N \rightarrow(N+1)}$, we adopt the linear relations between current and electric field, which are called Ohmic boundary conditions,

$$
\begin{gathered}
J_{0 \rightarrow 1}=\sigma E_{0}, \\
J_{N \rightarrow(N+1)}=\sigma E_{N} n_{N} .
\end{gathered}
$$

The relationship between the contact doping density and $\sigma$ in Eqs. (4) and (5) has been given by Xu et al. in Ref. 15.
For the SL samples investigated in Ref. 13, a typical contact conductivity value is $\sigma \approx 0.5$, under which the SL system is in the stationary regime. In the present work, we will study the DVB region, which is in the transition process from stationary to oscillation regime. As discussed in Sec. I, $\sigma$ should be less than 0.5 in order to observe DVBs. Our simulations show that the $\sigma$ value in the transition process ranges from 0.24 to 0.36 approximately. The exact transition values depend on the other SL parameters.

In the simulations, we use the fourth-order Runge-Kutta method to solve the SL system with the initial condition

$$
E_{i}(t=0)=\phi_{i} \quad \text { for } \quad i=0, \ldots, N .
$$

In this work, a uniform initial condition, $\phi_{i}=\phi$, is used. To understand the influence of the initial condition on the observed results, we also employ a random initial condition, where $\phi_{i}$ is a random number inside the interval $(\phi-0.2, \phi+$ 0.2 ). It is found that the simulation results do depend on the initial condition; however, the period branches observed are similar to that under the uniform initial condition. Therefore, the initial condition does not affect the phenomenon of period branches.

For $\sigma=0.5$, the static voltage branches obtained in our simulations by voltage up-sweeping from $\phi=0$ to high voltage values and voltage down-sweeping from high to low voltage values show a good agreement with Ref. 13 . Therefore, we believe that our simulation method is accurate enough.

\section{DISCRETE PERIOD BRANCHES}

As mentioned above, the dynamic voltage band was first observed in the transition process from static to dynamic regime by increasing the SL sample temperature. ${ }^{34}$ Here, we show that the coexistence of DVBs and SVBs could also be observed if the boundary contact conductivity $\sigma$ is varied from the static to dynamic regime. In Fig. 1, we plot the time-averaged current-voltage characteristics of the SL system given in Sec. II when $\sigma$ varies from 0.2 to 0.38 .

For large $\sigma(\sigma=0.38)$, the system is in the stationary regime and the $I-V$ curve exhibits a series of current branches corresponding to stationary EFD configurations. Three branches and a tiny part of the adjacent $I-V$ branch are shown by solid stars. With decreasing $\sigma$, the so-called DVBs (denoted by open symbols), within which self-sustained current oscillation can be observed, begin to emerge from the interface between two adjacent SVB branches (denoted by solid symbols). Two full DVB regions are denoted by $A$ and $B$ in Fig. 1. If $\sigma$ is further decreased, these DVBs will expand and even squeeze out the SVB region. At small $\sigma(\sigma=0.2$, shown by open squares in Fig. 1), the SL system comes into the oscillation regime, and the time-averaged $I-V$ curve is almost flat. These results resemble well the transition process when changing the SLs sample temperature in previous works.

We focus on the voltage dependence of the current self-oscillation period in the DVB region and choose an intermediate contact conductivity value, $\sigma=0.26$ (see Fig. 2). To evaluate the current oscillation period, we first record the current traces at a given $V$ and $\sigma$ value. Then, a series of periods are obtained by calculating the time interval between two successive maxima in the time traces (see the sharp current 


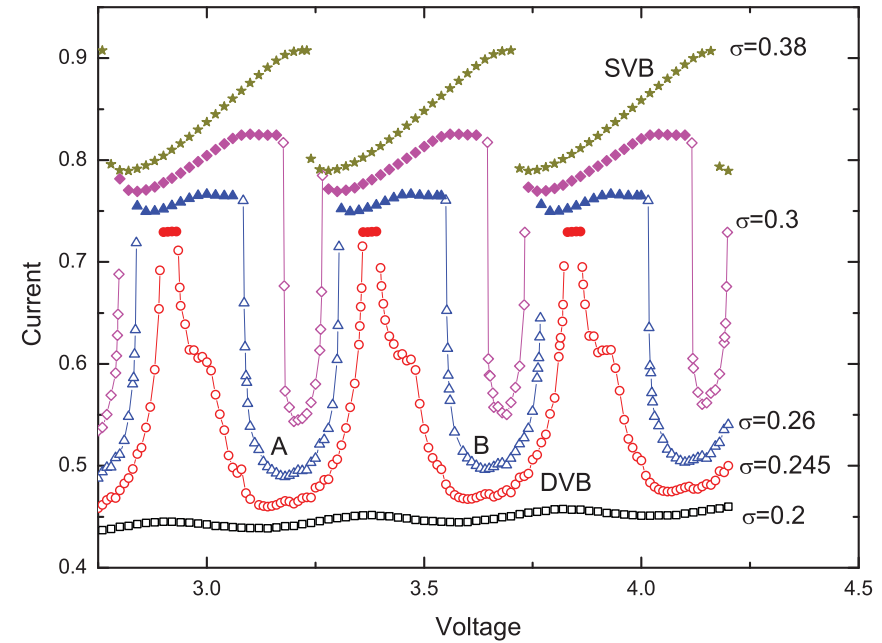

FIG. 1. (Color online) Time-averaged $I-V$ characteristics for differrent boundary contact conductivities. Open symbols indicate the DVB region, while solid symbols denote the SVB region. For the sake of clarity, the current values for $\sigma=0.26, \sigma=0.3$, and $\sigma=0.38$ are shifted up by $0.02,0.04$, and 0.06 , respectively.

peak in Fig. 3). The average of these periods is considered as the oscillation period at the given parameters. To ensure that the average period is accurate, the variance of these periods (not shown here) is also calculated and the results show that the variance is very small compared with the average value.

For DVB region $B$, which is enlarged in Fig. 2(b), the period-voltage curve consists of many discrete period branches. Two different types of period branches can be identified. The first type of branches (type I) are denoted by number 1, 2, 3, 4, 5, ... as shown in Fig. 2(b), and all these branches form a U-shaped curve in the DVB region. The length of type-I branches increases with increasing voltage. At the left (low-voltage) side of the U-shaped region, the branch length is so small that we have to enlarge the figure to see these branches [see the inset panel in Fig. 2(b)]. While at the right side, there is only one branch which is denoted by the number 1 . The second branch type (type II) contains only one branch, which is a tiny low-period branch denoted by the number 0 at the left boundary of the DVB region in Fig. 2(b). From our simulation results, we find that

(i) The right side of the U-shaped region is occupied by branch 1 , and the oscillation period increases toward infinity with increasing voltage.

(ii) At the left side of the U-shaped region, the current oscillation period increases toward infinity with increasing branch number.

(iii) For each type-I or type-II branch, the period increases as the voltage increases.

The U-shaped period curve in Fig. 2 agrees well with the results reported in previous works. ${ }^{35,38}$ Here, we observe two new phenomena: (1) A new period branch (branch number 0) appears at the left side of the DVB region. (2) It is shown that the U-shaped period curve is divided into many discrete branches. However, these branches appear only in a narrow voltage range $(V \approx 3.54 \rightarrow 3.57$ ), so they were not reported in the literature.
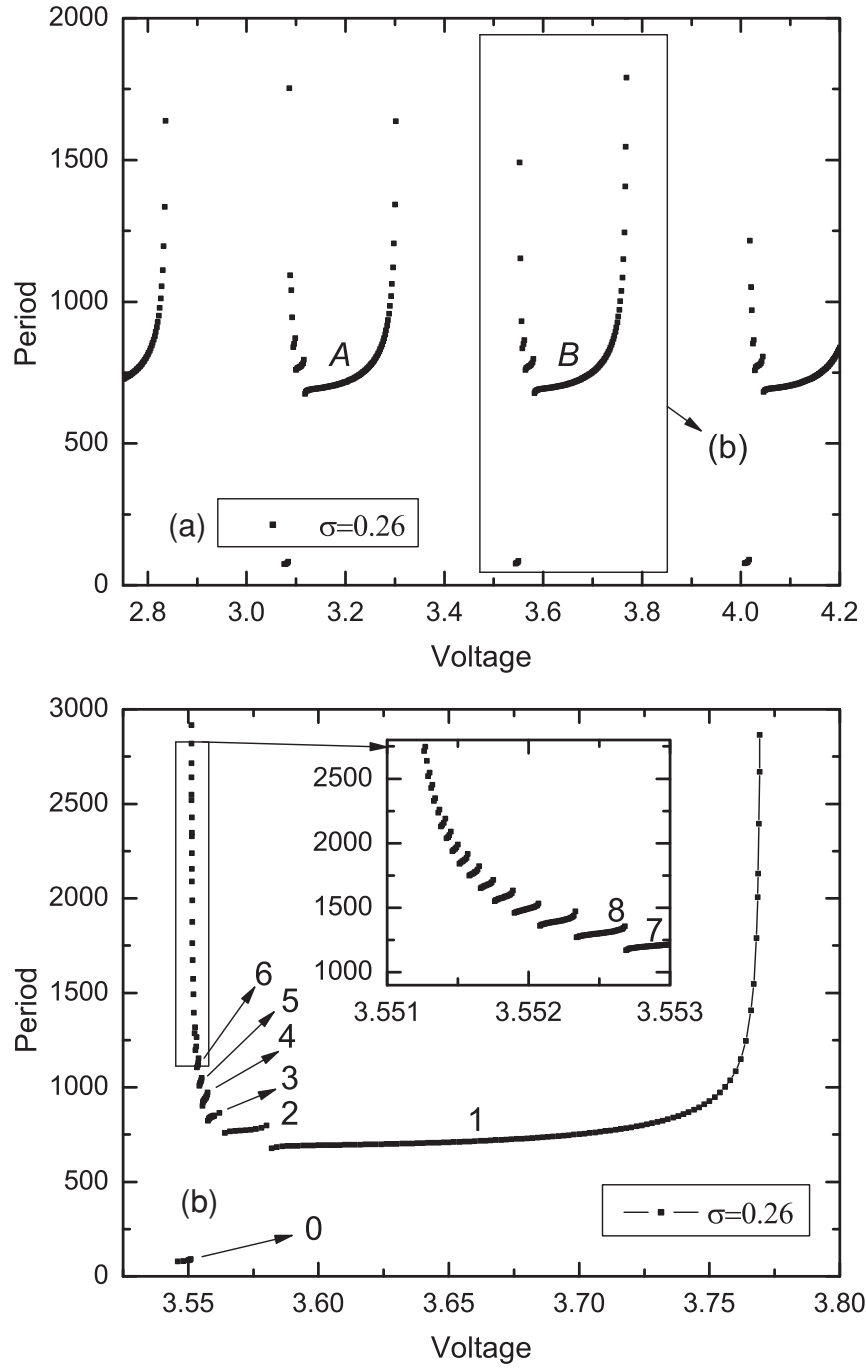

FIG. 2. (a) Voltage dependence of the self-sustained oscillation period in the dynamic voltage bands for $\sigma=0.26$. For the sake of clarity, the DVB region $B$ is enlarged in (b) and part of (b) is also enlarged in the inset figure.

\section{DIPOLE GENERATION PROCESS}

In order to understand the discrete period-voltage branches in the DVB region, it is necessary to study the time trace of the total current and the formation of the dipole at the emitter side of superlattices. First of all, we choose two points, $V=3.7$ at intermediate position and $V=3.768$ near the right boundary, from the first type-I branch denoted by 1 . The corresponding current traces $J(t)$ and the dynamic evolution of charge density patterns are plotted in Fig. 3. The current traces demonstrate a typical dipole-tripole scenario described in the previous works. ${ }^{13,33}$ In the time range $t \approx 12700 \rightarrow 13000$ for $V=3.7$, a dipole, which consists of a CAL (light region) and a CDL (dark region), travels across the SL with almost constant velocity. When the leading CDL reaches the collector side (SL well $i=40$ ) and disappears, the remaining CAL starts to slow down because of the total bias confinement. From the previous theoretical works on CAL wave fronts, ${ }^{16-18}$ the current value for CAL with low velocity should be higher than that for the CAL with high velocity, so the total current 

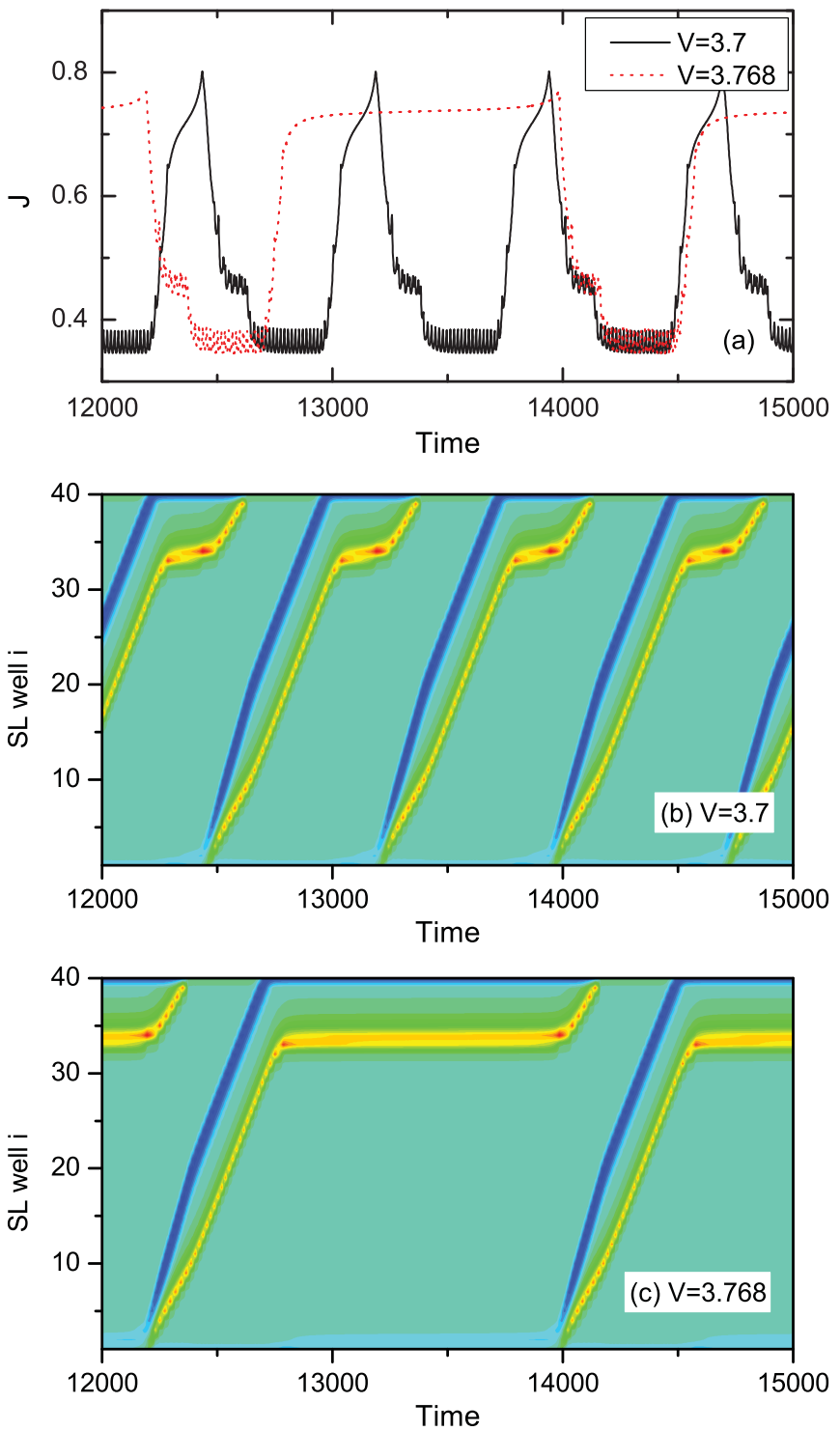

FIG. 3. (Color online) (a) The current traces at two applied voltage values in branch $1, V=3.7$ and $V=3.768$. (b) and (c) The dynamic evolution of charge density patterns for $V=3.7$ and $V=3.768$.

starts to increase when the CAL slows down. The increase of the current injects electrons into the SL system from the emitter side (SL well $i=1$ ), which will generate a charge dipole and push the old CAL to the collector side because of the total bias condition. Hence, as the system evolves, there are two CALs and one CDL (tripole) inside the SLs. When the old CAL reaches the collector side and vanishes, a new dipole process starts. Thus, a whole oscillation period is composed of a dipole-tripole process and a dipole generation process. Note that the time that the SLs spend in the dipole-tripole process is insensitive to the voltage, while it needs more time to generate a new dipole for larger voltages.

For the other type-I branches, such as 2 and 3, two intermediate points $V=3.57$ and $V=3.56$ are chosen. Figure 4 depicts the simulation results. It is found that the dipole-tripole scenario is very similar to that of branch 1 , but the formation processes of new dipoles are different.
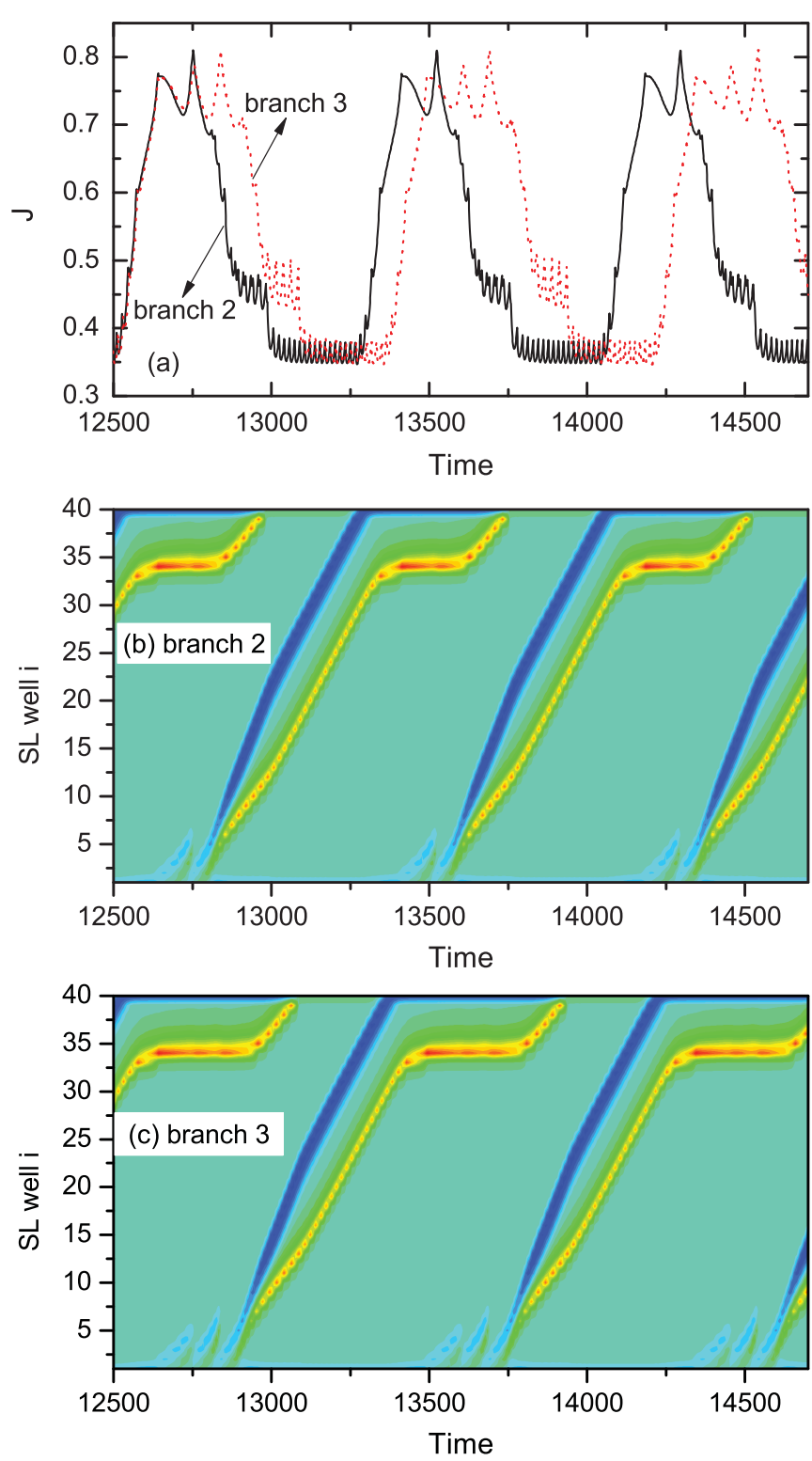

FIG. 4. (Color online) (a) The current traces at the intermediate points $(V=3.57$ and $V=3.56)$ of branches 2 and 3. (b) and (c) The dynamic evolution of charge density patterns for $V=3.57$ and $V=3.56$.

For branch 2, there exist two current peaks before the SL system assumes the dipole-tripole process. At the first current peak, a small dipole is created at the emitter side, but it fails to grow up completely. The new CAL and CDL travel through a couple of SL wells before they collide with each and die out [see Fig. 4(b)]. When the first dipole vanishes, another dipole is created, corresponding to the second current peak, and then the dipole-tripole process starts with the second dipole. As for branch 3, three dipoles are needed to develop a good dipole that can trigger the dipole-tripole scenario [see Fig. 4(c)]. In the same way, there are three current peaks in the time trace of the total current for branch 3.

Figure 5 plots the phase portraits in terms of electron densities in two neighboring SL wells at the emitter side, $i=1$ and $i=2$. Four branches (1, 2, 3, and 4) are selected and each 

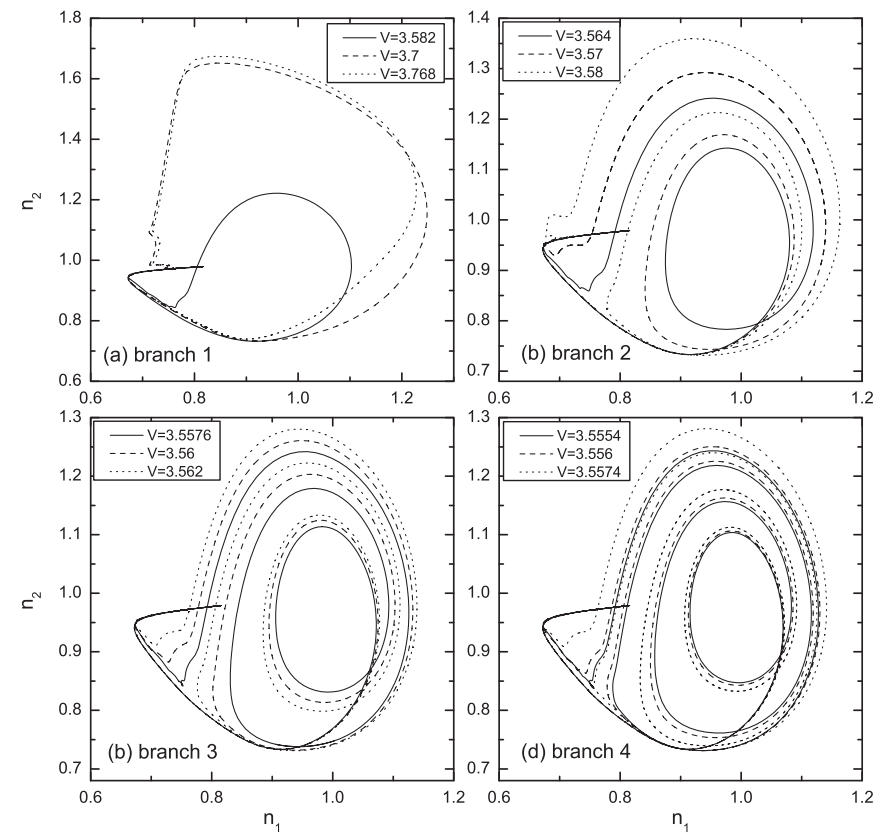

FIG. 5. The $n_{1}-n_{2}$ phase portraits for (a) branch 1, (b) branch 2 , (c) branch 3, and (d) branch 4. Solid line, dashed line, and dotted line represent the left boundary point, intermediate point, and right boundary point in each branch.

branch is studied at three points. The first point is near the left side of each branch. The second one is near the intermediate position, and the third one is on the right side. For branches 1 , 2 , and 3, the same points as Figs. 3 and 4 are used. If $n_{1}<n_{2}$, there should be a charge depletion layer at SL well $i=1$, and $n_{1}>n_{2}$ implies a charge accumulation layer. A circle around the neutral point $\left(n_{1}=n_{2}=1.0\right)$ in the phase portraits indicates that a dipole is created at the emitter side and then it moves into the superlattices. The inner circle denotes the small weak dipole which is not well developed, while the outermost circle represents a fully developed dipole.

In Fig. 5, it is shown that there are $k$ circles around the neutral point for the three points chosen in period branch $k$. It is confirmed that $k$ circles could be found in the $n_{1}-n_{2}$ phase portrait for all the points in branch $k$ (here, $k$ denotes the type-I branch, and $k=1,2,3,4)$. Further simulations confirm that this conclusion is also valid for branches of larger numbers. Actually, we just use the number of circles to mark different type-I branches.

From the time traces in Figs. 3 and 4, it is seen that the time period of the self-sustained current oscillation consists of two parts: the dipole-tripole process and the dipole-generation process. The self-oscillation period is insensitive to the time that the SL system spends in the dipole-tripole process, while the time needed to create a well developed dipole plays a dominant role for the oscillation period. This time depends on two factors: (1) how much time it needs to create a new dipole, and (2) how many dipoles can be generated.

For branch 1, only one dipole is created, but it needs increasing time to create a new dipole with increasing voltage, as shown in Fig. 3. Hence, the time period increases with increasing voltage [finding (i) in Sec. III]. The oscillation period increases significantly toward infinity when the voltage is close to the right boundary of the DVB region. The SL system will come into the stationary regime with a stationary domain wall located at SL well $i=33$ if the voltage exceeds the right threshold.

For the branches with number $k>1$, the time period increases with increasing branch number [finding (ii) in Sec. III] because a rising number of dipoles die out before the final dipole is well developed. As a result, increasing time is needed to generate more dipoles. The oscillation period will increase toward infinity with increasing branch number at the left side of the U-shaped region.

If the voltage decreases further, the SL system turns into the type-II branch denoted by the number 0 in Fig. 2, which is a simple low-period oscillation branch (see Fig. 6). In this case, a clear CAL could be found near the SL well $i=34$. At the emitter side, weak dipoles are generated periodically, but all these dipoles fail to develop completely. Thus, there is no dipole-tripole process in branch 0 . In other words, branch 0 actually is a limiting type-I branch with $k=\infty$. For branch 0 , the dipole generated at the emitter side becomes much weaker with decreasing voltage. Finally, a stationary domain wall is established at SL well $i=34$ and no dipole can be created anymore. Note that the position of the stationary domain wall moves one SL well from the left to right boundaries of DVB region $B$.

For fixed period branch number $k>1$, the number of dipoles that vanish inside the SLs remains unchanged (for the type-II branch, this number is infinity). At a high voltage, the length of the new generated dipoles (the distance between the new CAL and CDL) should be larger than that of a lower voltage condition because of the total bias confinement.
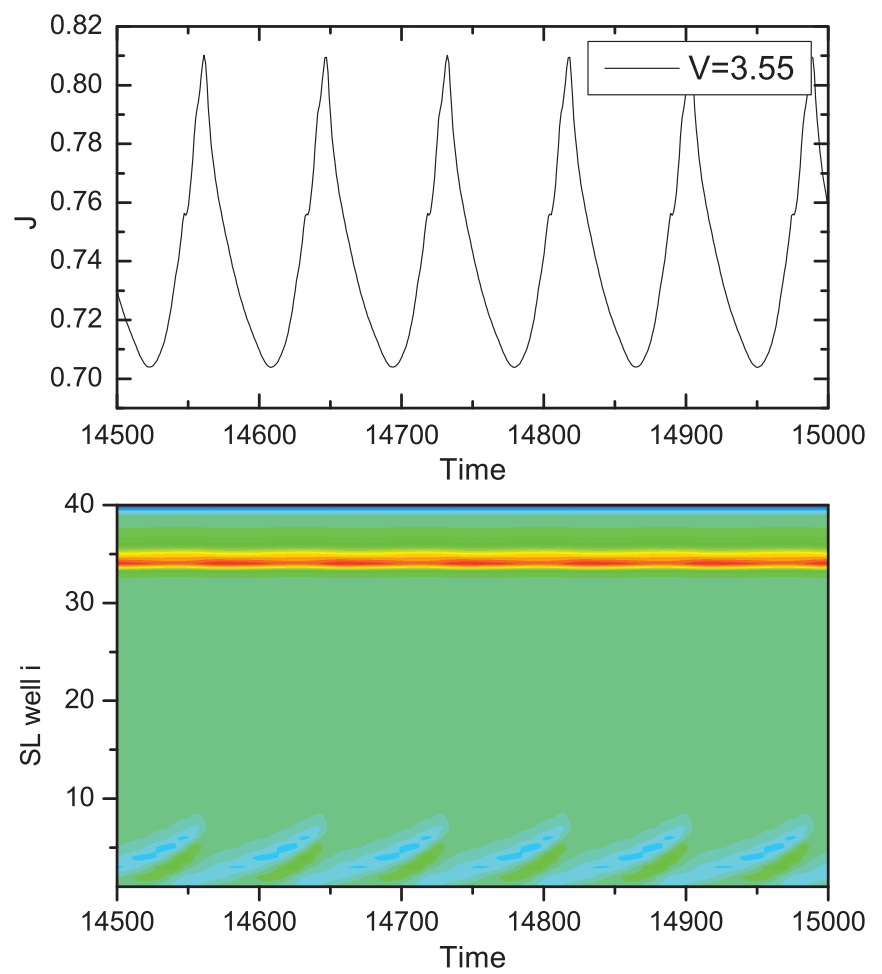

FIG. 6. (Color online) The current trace (a) and dynamic evolution of charge density pattern (b) at the intermediate point $(V=3.55)$ in the type-II branch 0 . 
So, the new dipoles under a higher voltage can survive a longer time before they disappear. That is why the self-oscillation period increases with increasing voltage in the same branch [finding (iii) in Sec. I]. However, this explanation cannot be applied to the dependence of the oscillation period on the voltage in branch 1 , because the increase of the oscillation period in branch 1 is due to the increasing time needed to create a new dipole.

In Ref. 38, it is reported that the self-oscillation begins and ends at supercritical Hopf bifurcations at the left and right boundaries of the DVB. On the other hand, saddle-node bifurcations are found at the sawtooth-like boundary of the $V-\sigma$ phase diagram. ${ }^{27}$ Because the left and right boundaries of the DVB region correspond to the left and right sides of the sawtooth-like boundary tongue, it is not clear what type of bifurcation will occur at the boundaries of the DVB.

In this work, it seems that the DVB region disappears via a supercritical Hopf and saddle-node bifurcation at the left and right boundaries, respectively. At the left DVB boundary (branch 0), the current oscillation amplitude shrinks to zero while the oscillation period remains finite with decreasing voltage, which indicates a supercritical Hopf bifurcation. At the right boundary (branch 1), the oscillation period goes to infinity when the current oscillation ends with a finite oscillation amplitude. This is a generic characteristic of saddle-node infinite period bifurcation with a homoclinic orbit. However, our simulation results are not sufficient to identify the bifurcation scenario at the DVB boundaries. Further investigations are needed to confirm our conclusions. This will be considered in future work.

\section{BISTABILITY BETWEEN DIFFERENT PERIOD BRANCHES}

In experiments, the $I-V$ characteristics are always obtained by voltage up-sweeping and down-sweeping. Bistability between different stable $I-V$ branches have been widely investigated in simulations and experiments. $1,2,19,20,40$ Figure 7(a) depicts the time-averaged $I-V$ curves in both sweep-up (squares) and sweep-down (triangles) directions in the DVB region $B$ shown in Fig. 2. The two insets in Fig. 7(a) show the enlargements of the hysteresis regions at the left and right boundaries, respectively. Similar bistability behavior in experiments has been reported in Ref. 40, where the bistability was observed by sweeping up and down the voltage. In Ref. 40, near the right boundary of the DVB region, it is found that the bistable region disappears via a subcritical Hopf bifurcation and saddle-node bifurcation in the voltage sweep-up and sweep-down directions, respectively. However, our simulation results show that the bifurcation scenarios at both the left and right DVB boundaries are not affected by the sweeping directions. This minor difference might be due to the different tunneling models adopted in our work and Ref. 40.

Figure 7(b) shows the voltage dependence of the current oscillation period in DVB region $B$. A part of the period branches are enlarged in the inset. It is found that bistability behavior can also be observed between different period branches, corresponding to the hysteresis between different current oscillation scenarios.
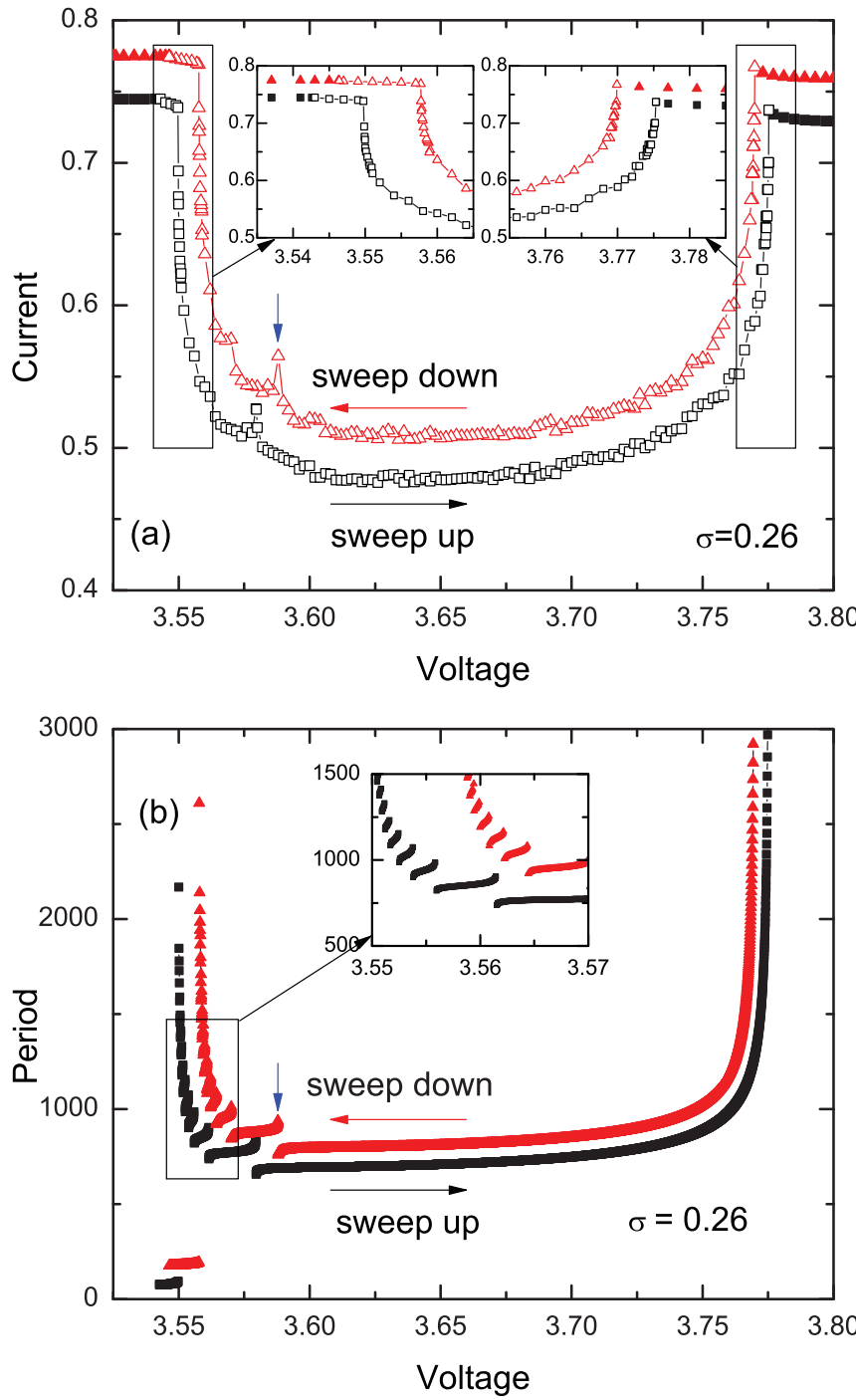

FIG. 7. (Color online) (a) Time-averaged $I-V$ curves in both sweep-up (squares) and sweep-down (triangles) directions in the DVB region $B$ at $\sigma=0.26$. Open symbols denote the DVB region and solid symbols represent stationary $I-V$ characteristics. (b) Voltage dependence of the current oscillation period corresponding to the DVB region $B$. The insets in (a) and (b) show enlargement of the hysteresis region. For the sake of clarity, the curves for downsweeping in (a) and (b) are shifted up by 0.03 and 100 , respectively.

Note that there exists a current peak on both up-sweeping and down-sweeping current curves, denoted by a vertical blue arrow in Fig. 7(a). This current peak corresponds to the transition from branch 1 to branch 2 [see the blue arrow in Fig. 7(b)]. Actually, if the $I-V$ curve is further enlarged, more current peaks in the $I$ - $V$ curve will emerge both in Figs. 2 and 7, corresponding to the transition points between adjacent period branches.

\section{CONCLUSIONS AND DISCUSSION}

In summary, the voltage dependence of the self-oscillation period in weakly coupled SLs is investigated in the DVB region. It is revealed that the period-voltage curve consists 
of two types of period branches depending on different dipole-generation processes at the emitter side. For the type-I branch, the time needed to generate a well developed dipole, which will trigger a dipole-tripole process, plays a dominant role for the self-oscillation period. Depending on how many dipoles can be generated at the emitter side, the period curve is divided into a series of branches. For the type-II branch, no dipole-tripole process is observed. Weak dipoles are created at the emitter side periodically and all die out after they travel forward for a couple of SL wells. Consequently, the oscillation period in this branch is very low. By voltage up-sweeping and down-sweeping, we observe the bistability between different branches of current oscillation period.

\section{ACKNOWLEDGMENTS}

This work was supported in part by the National Natural Science Foundation of China and the Hong Kong University of Science and Technology School Initiative Grant No. SBI07/08.EG01. We would like to thank the High Performance Cluster Computing Center at Hong Kong Baptist University for the use of its computing facilities. *mejwang@ust.hk

${ }^{1}$ L. L. Bonilla, J. Phys. Condens. Matter 14, 341 (2002).

${ }^{2}$ L. L. Bonilla and H. T. Grahn, Rep. Prog. Phys. 68, 577 (2005).

${ }^{3}$ A. Wacker, Phys. Rep. 357, 1 (2002).

${ }^{4}$ G. Platero and R. Aguado, Phys. Rep. 395, 1 (2004).

${ }^{5}$ L. Esaki and R. Tsu, IBM J. Res. Dev. 14, 61 (1970).

${ }^{6}$ L. L. Bonilla, J. Galán, J. A. Cuesta, F. C. Martínez, and J. M. Molera, Phys. Rev. B 50, 8644 (1994).

${ }^{7}$ L. Esaki and L. L. Chang, Phys. Rev. Lett. 33, 495 (1974).

${ }^{8}$ H. T. Grahn and R. J. Haug, W. Müller, and K. Ploog, Phys. Rev. Lett. 67, 1618 (1991).

${ }^{9}$ L. L. Bonilla, G. Platero, and D. Sanchez, Phys. Rev. B 62, 2786 (2000).

${ }^{10}$ A. Wacker, M. Moscoso, M. Kindelan, and L. L. Bonilla, Phys. Rev. B 55, 2466 (1997).

${ }^{11}$ J. Kastrup, R. Hey, K. H. Ploog, H. T. Grahn, L. L. Bonilla, M. Kindelan, M. Moscoso, A. Wacker, and J. Galán, Phys. Rev. B 55, 2476 (1997).

${ }^{12}$ H. T. Grahn, H. Schneider, and K. von Klitzing, Phys. Rev. B 41, 2890 (1990).

${ }^{13}$ L. L. Bonilla, R. Escobedo, and G. Dell'Acqua, Phys. Rev. B 73, 115341 (2006).

${ }^{14}$ A. Amann, A. Wacker, L. L. Bonilla, and E. Schöll, Phys. Rev. E 63, 066207 (2001).

${ }^{15}$ H. D. Xu and S. W. Teitsworth, Phys. Rev. B 76, 235302 (2007).

${ }^{16}$ A. Carpio, L. L. Bonilla, A. Wacker and E. Schöll, Phys. Rev. E 61, 4866 (2000).

${ }^{17}$ A. Carpio, L. L. Bonilla, and G. Dell'Acqua, Phys. Rev. E 64, 036204 (2001).

${ }^{18}$ J. Wang and Z. G. Zheng, Chin. Phys. B 17, 4129 (2008).

${ }^{19}$ Y. H. Zhang, R. Klann, and K. H. Ploog, Appl. Phys. Lett. 70, 2825 (1997).

${ }^{20}$ K. J. Luo, S. W. Teitsworth, H. Kostial, H. T. Grahn, and N. Ohtani, Appl. Phys. Lett. 74, 3845 (1999).

${ }^{21}$ X. R. Wang and Q. Niu, Phys. Rev. B 59, 12755 (1999).
${ }^{22}$ Z. Z. Sun, H. T. He, J. N. Wang, S. D. Wang, and X. R. Wang, Phys. Rev. B 69, 045315 (2004).

${ }^{23}$ D. Sánchez, M. Moscoso, L. L. Bonilla, G. Platero, and R. Aguado, Phys. Rev. B 60, 4489 (1999).

${ }^{24}$ G. K. Rasulova, P. N. Brunkov, A. Y. Egorov, and A. E. Zhukov, J. Appl. Phys. 105, 033711 (2009).

${ }^{25}$ G. Yang, H. Meng, L. F. Zhang, and S. P. Zhou, J. Appl. Phys. 103, 123701 (2008)

${ }^{26}$ Z. Z. Sun, K. L. Chen, H. T. He, J. N. Wang, Y. Q. Wang, and X. R. Wang, New J. Phys. 6, 148 (2004).

${ }^{27}$ J. Hizanidis, A. Balanov, A. Amann, and E. Schöll, Phys. Rev. Lett. 96, 244104 (2006)

${ }^{28}$ Y. Zhang, J. Kastrup, R. Klann, K. H. Ploog, and H. T. Grahn, Phys. Rev. Lett. 77, 3001 (1996).

${ }^{29}$ K. J. Luo, H. T. Grahn, K. H. Ploog, and L. L. Bonilla, Phys. Rev. Lett. 81, 1290 (1998).

${ }^{30}$ O. M. Bulashenko and L. L. Bonilla, Phys. Rev. B 52, 7849 (1995).

${ }^{31}$ K. J. Luo, H. T. Grahn, S. W. Teitsworth, and K. H. Ploog, Phys. Rev. B 58, 12613 (1998).

${ }^{32}$ A. Amann, K. Peters, U. Parlitz, A. Wacker, and E. Schöll, Phys. Rev. Lett. 91, 066601 (2003).

${ }^{33}$ A. Amann, J. Schlesner, A. Wacker, and E. Schöll, Phys. Rev. B 65, 193313 (2002)

${ }^{34}$ J. N. Wang, B. Q. Sun, X. R. Wang, Y. Q. Wang, W. K. Ge, and H. L. Wang, Appl. Phys. Lett. 75, 2620 (1999).

${ }^{35}$ X. R. Wang, J. N. Wang, B. Q. Sun, and D. S. Jiang, Phys. Rev. B 61, 7261 (2000).

${ }^{36}$ C. Y. Li, B. Q. Sun, D. S. Jiang, and J. N. Wang, Semicond. Sci. Technol. 16, 239 (2001).

${ }^{37}$ H. T. He, Z. Z. Sun, X. R. Wang, Y. Q. Wang, W. K. Ge, and J. N. Wang, Solid State Commun. 136, 572 (2005).

${ }^{38}$ D. Sanchez, L. L. Bonilla, and G. Platero, Phys. Rev. B 64, 115311 (2001).

${ }^{39}$ H. T. He, Y. Q. Wang, W. K. Ge, and J. N. Wang, Semicond. Sci. Technol. 23, 085003 (2008).

${ }^{40}$ H. T. He, Y. Q. Wang, W. K. Ge, and J. N. Wang, New J. Phys. 10, 033019 (2008). 